\title{
BMJ Open HOspitals and patients WoRking in Unity (HOW R U?): protocol for a prospective feasibility study of telephone peer support to improve older patients' quality of life after emergency department discharge
}

\begin{abstract}
Judy A Lowthian, ${ }^{1}$ Alyse Lennox, ${ }^{1}$ Andrea Curtis, ${ }^{1}$ Jeremy Dale, ${ }^{2}$ Colette Browning, ${ }^{3,4,5}$ De Villiers Smit, ${ }^{6}$ Gillian Wilson, ${ }^{7}$ Debra O'Brien, ${ }^{8}$ Cate Rosewarne, ${ }^{9}$ Lee Boyd,${ }^{10}$ Cath Garner, ${ }^{11}$ Peter Cameron ${ }^{1,6}$
\end{abstract}

To cite: Lowthian JA, Lennox A, Curtis $A$, et al. HOspitals and patients WoRking in Unity (HOW R U?): protocol for a prospective feasibility study of telephone peer support to improve older patients' quality of life after emergency department discharge. BMJ Open 2016:6:e013179. doi:10.1136/bmjopen-2016013179

- Prepublication history for this paper is available online. To view these files please visit the journal online (http://dx.doi.org/10.1136/ bmjopen-2016-013179).

Received 24 June 2016 Revised 10 November 2016 Accepted 14 November 2016

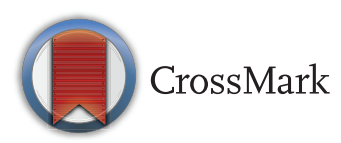

For numbered affiliations see end of article.

Correspondence to Dr Judy Lowthian, Judy.Lowthian@monash.edu

\section{ABSTRACT}

Introduction: Older people presenting to an emergency department (ED) have a higher likelihood of social isolation, Ioneliness and depression; which are all associated with negative health outcomes and increased health service use, including higher rates of ED attendance. The HOW $R U$ ? study aims to ascertain the feasibility and acceptability of a postdischarge telephone support programme for older ED patients following discharge. The intervention, which aims to improve quality of life, will be delivered by hospitalbased volunteers.

Methods and analysis: A multicentre prospective uncontrolled feasibility study will enrol 50 communitydwelling patients aged $\geq 70$ years with symptoms of Ioneliness or depression who are discharged home within 72 hours from the ED or acute medical ward. Participants will receive weekly supportive telephone calls over a 3-month period from a volunteerpeer. Feasibility will be assessed in terms of recruitment, acceptability of the intervention to participants and level of retention in the programme. Changes in level of loneliness (UCLA-3 item

Loneliness Scale), mood (Geriatric Depression Scale- 5 item) and health-related quality of life (EQ-5D-5L and EQVAS) will also be measured postintervention (3 months).

Ethics and dissemination: Research ethics and governance committee approval has been granted for this study by each participating centre (reference: 432/15 and 12-09-11-15). Study findings will inform the design and conduct of a future multicentre randomised controlled trial of a postdischarge volunteer-peer telephone support programme to improve social isolation, Ioneliness or depressive symptoms in older patients. Results will be disseminated through peer-reviewed journal publication, and conference and seminar presentation. Trial registration number: ACTRN12615000715572, Pre-results.

\section{Strengths and limitations of this study}

This is the first study to examine volunteer-peer telephone support for discharged older emergency patients.

- We will evaluate (1) feasibility of recruitment delivery of the intervention and outcome measure ascertainment at study conclusion; (2) intervention acceptability and retention; and (3) changes in level of loneliness, depressive symptoms and quality of life.

- The feasibility study design is not powered to determine intervention effectiveness.

- Results will inform the design and conduct of a future multicentre randomised controlled trial of postdischarge volunteer-peer telephone support to improve health outcomes in older emergency patients.

\section{BACKGROUND}

Older people aged $\geq 70$ years are an evergrowing emergency department (ED) population, with attendances accelerating at a rate beyond that expected from demographic change alone. ${ }^{2} 2$ They are the highest users of EDs, ${ }^{13}$ are four times more likely to reattend within 12 months than those $<70$ years of age ${ }^{3}$ and more likely to be admitted to hospital. ${ }^{4}$ This older ED population have a high likelihood of social isolation, loneliness, lack of social support ${ }^{5}{ }^{6}$ and depressive feelings. ${ }^{7} 8$ Feeling depressed is associated with higher rates of ambulance use and ED attendance. $^{9}{ }^{10}$ Social isolation is also associated with a fourfold to fivefold increase in the likelihood of representation and admission to hospital within 12 months. ${ }^{11}$ In 
addition, feeling sad or depressed is an independent predictor of early and frequent reattendance to ED by older patients, after controlling for medical history and diagnosis. ${ }^{12}$ This propensity to reattend must be reduced, as an ED visit is described as a sentinel event in older age ${ }^{13}$ with associated functional decline, admission to nursing care facilities and death in subsequent months. ${ }^{6} 1415$

Social isolation, loneliness and depressed mood are distinct entities that are prevalent among older community-dwellers, with research indicating that:

- seventeen per cent of older people have contact with family, friends or neighbours less than weekly, and $11 \%$ have contact less than monthly; ${ }^{16}$

- television is the main company for $40 \%$ of older people; ${ }^{17}$

- twelve per cent of the population aged $\geq 65$ years feel socially isolated $;^{18}$

- loneliness among older community dwellers is as high as $50 \%$ in the UK and Australia; ${ }^{19} 20$

- self-reported depression ranges from $6 \%$ to $20 \%$ in older Australian community-dwellers. ${ }^{21}$

These self-reported rates probably under-represent true levels because of an associated stigma among older people that such feelings are a character weakness, and that 'one should be able to cope or pull themselves together, ${ }^{21}$ Therefore, older patients who feel lonely or depressed are highly likely not to be identified, ${ }^{22}$ thus reducing the opportunity for appropriate support being implemented in the community.

Importantly, social isolation and loneliness are associated with negative health outcomes and lower health-related quality of life, as summarised in box $1 .^{23}$

\section{A potential solution}

HOspitals and patients WoRking in Unity (HOW R U? ) is a peer support programme for community-dwelling older people with symptoms of depression, social isolation or loneliness after discharge from the ED. The intervention is innovative as it is delivered by hospitalbased volunteers over the telephone.

Peer support is the "provision of knowledge, experience, emotional or practical help by someone sharing common characteristics, ${ }^{35}$ The social support model postulates that social relationships promote health and well-being; thus, peer support is hypothesised to reduce feelings of social isolation and loneliness, thereby improving well-being. ${ }^{36}$ Peer support is usually provided by a person sharing common characteristics, for example, age, gender, ethnicity or experience of illness. Equivalent 'status' between peer and patient is a feature of peer support that facilitates a high level of empathy delivered in a nonconfrontational manner. ${ }^{37}$

The telephone is increasingly used to deliver healthcare advice and support for patients. Most older people have telephone access and are likely to accept telephone-mediated peer support. Telephone-based peer support can be a satisfactory substitute for
Box 1 Health outcomes associated with social isolation, Ioneliness and depressive symptoms in older people

Social isolation and lack of social support

- Impact on early mortality is equivalent to smoking $>15$ cigarettes/day or being an alcoholic, with socially connected people $50 \%$ more likely to survive than those who are socially isolated (meta-analysis, 148 observational studies, $\mathrm{N}=308849$, mean age 64 years); ${ }^{24}$

- Excess risk of incident stroke in community-dwellers. ${ }^{25}$

Loneliness

- Increased risk of high blood pressure over a 4-year period; ${ }^{26}$

- Greater risk of cognitive decline and poorer cognitive function in older age $\mathrm{e}^{27-29}$ as well as a $64 \%$ increased chance of developing dementia; ${ }^{30}$

- Predictive of suicide in older age together with social isolation; ${ }^{31}$

- Predictor of functional decline and death. ${ }^{32}$

Depressive symptoms

- Increased risk of incident dementia; ${ }^{33}$

- Development of coronary heart disease and total mortality. ${ }^{34}$

face-to-face interaction, and many people prefer the relative anonymity and increased privacy of talking over the phone. ${ }^{38}$ Evaluation of the UK Call in Time telephone befriending service for older people indicated a major impact on their quality of life. ${ }^{39}$ Participants reported they felt a sense of belonging, valued knowing 'there's a friend out there' and that the service had a positive impact on their health and well-being, with increased self-confidence and alleviation of previous loneliness and anxiety.

Dale et $a l \mathrm{~s}^{37}$ updated Cochrane Review investigated the effects of peer support telephone calls for improving physical, psychological, behavioural and other health outcomes in 14 randomised controlled trials (RCTs) involving 8040 participants. Positive results were found in eight studies, with peer support effective in reducing depressive symptoms in new mothers, supporting breast feeding, promoting mammography screening, improving diabetes outcomes and colonoscopy screening. Peer support programmes have also been shown to reduce healthcare service use by older people, including admissions to hospital, nursing care facility admissions and community doctor visits. ${ }^{40}$

Peers may be volunteers who are trained to support and listen, but not to give medical advice or judgement. Their non-medical status helps to overcome any reluctance that patients may have in discussing feelings of loneliness or isolation. ${ }^{41}$ Hospital volunteers are trained to help support patients and families during an acute hospital admission or ED visit, which is a form of 'peer' support. With additional training and support, this volunteer role could be transferred beyond the hospital walls, to provide older patients with telephone peer support and social contact following discharge from the ED. 


\section{OBJECTIVES}

The current study will test the acceptability and feasibility of HOW R U? an intervention designed to support older vulnerable patients after hospitalisation. We hypothesise that:

1. it is feasible to enrol older patients aged $\geq 70$ years at the time of ED attendance, execute study procedures and measure functional outcomes in a multicentre observational study of a supportive volunteer-peer telephone intervention, to inform an RCT;

2. HOW R U? will be acceptable to patient and volunteer participants; and

3. there will be positive changes in the functional outcomes measured.

\section{METHODS AND ANALYSIS}

Study design and setting

$H O W R U$ ? is a pragmatic uncontrolled study testing the feasibility of a volunteer-peer telephone-support programme for older patients discharged from two EDs, short-stay units (SSUs) and acute medical wards (AMWs) at The Alfred and Cabrini Hospitals, both of which are large tertiary referral providers of healthcare that service public and private hospital patients in the inner southeastern suburbs of Melbourne, Australia. Recruitment started at The Alfred in November 2015 and is ongoing at Cabrini until August 2016.

\section{Participants}

Community-dwelling men and women aged $\geq 70$ years will be recruited.

Inclusion criteria: Patients who screen positive for symptoms of social isolation, depression and/or loneliness using the Social Isolation Index $(\mathrm{SII} \geq 2),{ }^{35}$ Geriatric Depression Scale-5 items (GDS-5 $\geq 2)^{42}$ and 3-item Loneliness Scale (UCLA-3 $\geq 6) ;{ }^{43}$ and are discharged home within 72 hours will be eligible for study inclusion.

Exclusion criteria: Patients will be excluded if they are triaged as category 1 level of urgency in ED; require surgery; live in a nursing care home; receive end-of-life care or are likely to be approaching end-of-life within 12 months using the Supportive Care and Palliative Care Indicators Tool criteria; ${ }^{44}$ have moderate to severe cognitive impairment using the Mini-Mental State Examination $(\mathrm{MMSE}<20)$ or telephone version (ALFI-MMSE $<16),{ }^{45} \mathrm{a}$ confirmed diagnosis of dementia or severe mental illness such as schizophrenia or psychosis; or are unable or unwilling to communicate in English by telephone.

Determining eligibility for participation will be a multistage process, established by the recruitment staff during medical record review and review of the completed screening questionnaires. Rates of interest, eligibility and consent will be monitored to assess intervention uptake.

\section{Recruitment and consent}

Recruitment will take place in the EDs, SSUs and AMWs during weekdays, 08:30-13:00. Study recruitment nurses will liaise with ED allied health and nurse team leaders at the beginning of each shift to identify potential participants based on the inclusion criteria. Participants will not be approached until after their clinical needs have been addressed, as these must be respected first and foremost, over and above their participation in this study. Potential participants will be screened by the recruitment staff for social isolation, loneliness and depressed mood, as well as for cognitive impairment; and eligible patients will be invited to participate. Written informed consent will be sought at the time of recruitment and confirmed at the first volunteer telephone call to reduce drop-out during the study.

If potentially suitable patients have been discharged from the ED or AMW, recruitment nurses will follow them up by telephone, to discuss the project and seek interest in participation in order to optimise recruitment numbers. Consent to notify the general practitioner (GP) of involvement in the study will also be sought from the participant.

\section{Sample size}

The study aims to establish the feasibility of recruitment, retention, assessment procedures, execution of the study protocol and testing intervention acceptability and adherence. A sample size of 50 participants across 2 hospital sites was selected, based on the pragmatics of recruitment and the necessities for examining feasibility. ${ }^{46}$

\section{Intervention}

The HOW R $U$ ? intervention has been designed in consultation with potential consumers, hospital volunteers and clinicians. It comprises:

1. routine screening for social isolation, loneliness and/ or depressive feelings in older users of the ED at the index ED attendance or hospital admission; followed by

2. weekly telephone support calls from a hospital volunteer (peer support person) for 3 months following $\mathrm{ED}$ and/or hospital discharge; and

3. referral to community-based services for ongoing support following the end of the study as needed.

An experienced older age hospital-based volunteer will be paired with a patient, matched by their preferred language. Patients will receive one telephone-support call every week over a 3-month period (up to 12 calls). The aim of the telephone calls is to provide emotional and social support. The first call will occur within 72 hours of discharge from ED, SSU or AMW.

The phone calls will focus on encouraging and supporting the patient and providing social stimulation and informal guidance about strategies that patients feel would improve their well-being, such as better self-care and/or social engagement with family, friends or community groups. Each call will be unstructured and patient-directed; however, the volunteer will ask the patient to describe any changes since the previous call, 
the outcomes of any planned actions and agree on new social goals. This model has been used by social service providers for older people in the community with positive outcomes, ${ }^{40}$ but is yet to be trialled in a cohort following hospital discharge.

The telephone calls will be conducted from the respective hospitals in an area allocated by the Volunteer Services Manager. HOW R U? volunteers will be provided with a summary of their participants' baseline data collection forms prior to conducting the first phone call.

In order to understand the impact of the various components of the intervention, telephone activity logs will be maintained by the volunteer for each participant. These logs will record the dates, times and length of each call, and the number of attempts required to make contact with the participant on each occasion. The content of the calls made will be described using a simple template. Participant drop-outs and their reasons for doing so will also be recorded on the activity log.

Volunteer Training: HOW R U? volunteer peers will be recruited from the hospital Volunteer Service. All volunteers will have participated in their respective hospitalbased volunteer training programme, which include workshops on confidentiality and privacy; rights and responsibilities in healthcare; professional and personal boundaries as a volunteer; emotions and responses; stress and self-care and communication and listening techniques. HOW $R \quad U$ ? volunteers will be actively involved in the regular provision of inhospital social and emotional support to acutely unwell patients and families.

At study initiation, the HOW $R U$ ? volunteers will attend a compulsory 4-hour project orientation session, including an overview of peer support; general information about mental health and ageing; expectations of their role; empathic listening techniques; policies and procedures; confidentiality and boundaries; risk management strategies and formal and informal community resources available to patients.

\section{Safety considerations}

It is not anticipated that $H O W R U$ ? will cause any specific harm or discomfort. If participants wish to terminate an individual telephone call or cease their involvement in the study, they may do so at any time, without any interference to any care provided by their treating hospital.

If a volunteer is concerned about the physical or mental health of a participant, the volunteer will liaise directly with the relevant Emergency Physician Coinvestigator. The concern will then be triaged and the participant's GP may be contacted, as required.

Volunteers will have access to their respective Volunteer Services Manager and the Chief Investigator for advice and support throughout the study. A monthly meeting of volunteers with the Chief Investigator and the Volunteer Services Managers will enable debriefing of any issues or concerns that arise.

Care transition at study end: Three main pathways have been developed for preparing participants for care transition from the project. Discussions will start at week 8 of the 12-week intervention, or earlier as needed. Participants will either: continue the telephone support calls if a mutual agreement is reached between the patient and the volunteer; be referred and transferred to an alternative community support programme for support or cease telephone support calls. If any issues arise for participants or volunteers as a result of planning the transition pathway, they will be referred to the Chief Investigator or their respective Volunteer Services Manager. If the participant would like to continue receiving calls from the volunteer but the volunteer has neither the time nor the inclination to continue, we will endeavour to find an alternative volunteer or arrange for continued support through a community-based agency.

\section{Data collection}

Baseline data will be collected from the patient during the initial ED visit, either in the ED or AMW, or over the telephone within 48 hours of discharge. Recruitment staff will collect biosociodemographic details, including age, gender, contact details, marital status, residential status, carer status, GP status, GP contact details, pet ownership, use of health services within the previous 12 months, current use of community services and comorbid health conditions. Standardised measurement instruments that demonstrate good psychometric properties and are used in older community-dwelling patient research will be applied, including: Social Isolation Index; ${ }^{35}$ UCLA-3-item Loneliness Scale $;{ }^{43}$ Geriatric Depression Scale 5-item (GDS-5); ${ }^{42}$ EQ-5D-5L, which measures perceived health-related quality of life in the dimensions of mobility, self-care, usual activities, pain/ discomfort and anxiety/depression; ${ }^{47}$ and the EQ visual analogue scale (EQ VAS), which is a visual analogue scale (0-100) measuring current health-related quality-of-life state. ${ }^{47}$ All instruments have been validated for use over the telephone.

\section{Outcomes}

Outcome data will be collected at 3 months after the initial ED presentation, via telephone by the Outcome Assessor. The primary outcomes are feasibility of study processes, and acceptability of the intervention to patient and volunteer participants.

This will include measurement of recruitment, assessment procedures, execution of the study protocol, how helpful the intervention was and the level of participation and retention in the intervention. Indepth, semistructured telephone interviews will be conducted at the end of the intervention, to enable patient participants to speak freely about their experiences and perceptions. A topic guide based on the Peer Support Evaluation 
Inventory ${ }^{48}$ will be used to provide prompts of key issues for exploration, including participants' experience of calls and their value, views about the programme, benefits of the programme and suggestions to enhance the effectiveness of $H O W R U$ ?

Two focus groups will be conducted with volunteer peers at the end of the intervention to allow the opportunity for interaction between volunteer peers to explore their experiences and perceptions. A topic guide based on the Peer Volunteer Experience Questionnaire ${ }^{48}$ will be used to provide prompts of key issues for exploration, including the experience of delivering the intervention, the impact of helping the participants on their own emotional well-being and their views about what might be needed to enhance the effectiveness of HOWR $U$ ?

Secondary outcomes include measurement of changes in perceived social isolation, level of loneliness, depressive symptoms and quality of life as measured by the SII, UCLA-3-item Loneliness Scale, GDS-5, EQ-5D-5L and EQ VAS, after completion of the intervention.

\section{Data management}

All data collected at baseline and during the 3-month outcome assessment telephone call will be recorded on a data collection form and will be labelled with a project-specific ID for each participant. The privacy of individuals is of paramount importance, and all identifiers will be removed prior to the data being analysed in an aggregated form. A telephone activity $\log$ will be maintained by the volunteers for each telephone call. Random audits of the telephone calls and the activity logs will ensure fidelity of the intervention and that a standard approach is being used. Following electronic data entry, a random selection of $10 \%$ of the data collection form and telephone activity log paper-based copies will be reviewed, to monitor data entry accuracy.

\section{Analysis}

Feasibility of conducting study processes will be assessed, including the volunteer-peer training programme and materials, eligibility screening and recruitment strategies, telephone call regime, risk management procedures and level of support required by the volunteers.

Intervention acceptability to patients and volunteers will be measured through rates of uptake by eligible patients, and retention in the intervention; alongside patient and volunteer feedback interviews which will consider acceptability from the perspectives of the volunteer and patient participants. Interview data will be analysed using a qualitative thematic framework approach. ${ }^{49}$ Data will be systematically scrutinised, charted and sorted into recurrent themes. Patterns and connections within the data will be highlighted in order to develop a framework of themes which will then be applied to the data. Commonalities and variations within and between participant groups (patient and volunteer participants) will be explored. Two researchers will perform the analysis independently, prior to discussing the emerging framework.

Preintervention and postintervention scores of social isolation, mood, loneliness and health-related quality of life will be analysed using paired t-tests to compare any differences with a significance level of $\mathrm{p}=0.05$.

The study findings will inform the design and conduct of a future multicentre RCT of a postdischarge volunteer-peer telephone support programme to improve social isolation, loneliness or depressive symptoms in older patients.

\section{DISSEMINATION}

The study Steering Committee will provide overall trial supervision. Written informed consent will be sought from all participants for their participation and the publication of the results. Confidentiality is of paramount importance, and the volunteer-peer supporters are bound by hospital guidelines to maintain professional behaviour, with adherence to patient confidentiality regulations at all times. Participants will be reminded that they are free to withdraw at any time, and that their data will be stored securely and anonymously. All data will be stored on a secure password-protected university server and archived for 7 years after study completion. The results will be disseminated through peer-reviewed journal publication, and conference and seminar presentation, whereby it will not be possible to identify participants.

\section{DISCUSSION}

Older people are a significant proportion of ED attendances. ${ }^{1-3}$ Many lack social support and have symptoms of loneliness, social isolation and/or depression; $;^{6} 5051$ all of which are associated with negative health outcomes, functional decline, institutionalisation, mortality and increased hospital use. ${ }^{7112352}$ Furthermore, with population ageing, it is likely that the number of older people at risk of social isolation and loneliness will continue to grow, as will their rates of ED use.

Social isolation, loneliness and depressive symptoms are not routinely screened for during ED attendances or short hospital admissions other than in research settings. Targeted management of older people suffering from social isolation, loneliness or depressive symptoms is effective with improving symptoms. ${ }^{52}$ Therefore, systematic identification of social isolation, loneliness or depressive symptoms at the time of ED attendance alongside postdischarge support should help combat the associated negative consequences, and diminish this important public health and individual burden.

This paper describes the protocol for a pragmatic, observational study to examine the feasibility and acceptability of providing volunteer-peer telephone support for this vulnerable population. Our overarching hypothesis is that volunteer-peer telephone-support will help reduce symptoms of social isolation, loneliness and 
Figure 1 Hypothesised effects of HOW R U?

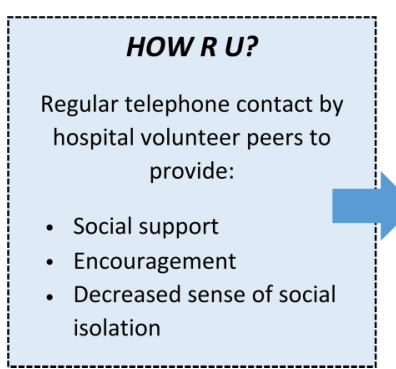

\section{PATIENT EFFECTS}

- Increased perceived social support

- Reduced sense of loneliness Improved mood

- Improved health-related

quality of life

- Improved patient experience
HEALTH SYSTEM EFFECTS

- Decreased ambulance use

- Decreased ED re-

attendance

- Decreased hospital

admission depressive feelings, through an improvement in mood and quality of life; and these effects will be associated with a reduction in the rate of return ED visits and hospital readmissions (figure 1).

$H O W R U$ ? has the potential to improve quality of life for older people in the community. It will also raise awareness of mental health issues in older people by GPs, health workers and family, and help redirect older people with symptoms of depression, loneliness and social isolation to appropriate services in a timely way. This will facilitate closer relationships between hospitals and their communities. Secondary benefits include the positive effects that the act of meaningful volunteering has on the peer supporter; with a positive correlation between volunteering and perceived health, and a negative correlation to depression in older volunteers. ${ }^{53}$ Volunteers represent a significant adjunct resource for meeting some of the health and social care service needs of our more vulnerable older population; as well as being inexpensive, which is an important consideration, given the financial constraints of health systems across the world.

The quantitative and qualitative findings of this feasibility study will be used to inform further development of the HOW R $U$ ? intervention and its mode of delivery, as well the design and development of a future RCT and programme evaluation, which will test the effectiveness of HOW R U? compared with usual care in improving quality of life, through improvement of symptoms of depression, social isolation and loneliness; and in reducing reattendances and hospitalisations.

\author{
Author affiliations \\ ${ }^{1}$ School of Public Health and Preventive Medicine, Monash University, \\ Melbourne, Victoria, Australia \\ ${ }^{2}$ Warwick Medical School, University of Warwick, Coventry, UK \\ ${ }^{3}$ RDNS Institute, RDNS, Melbourne, Victoria, Australia \\ ${ }^{4}$ International Institute for Primary Health Care Research, Shenzhen, China \\ ${ }^{5}$ School of Primary Health Care, Monash University, Melbourne, Victoria, \\ Australia \\ ${ }^{6}$ Emergency and Trauma Centre, Alfred Health, Melbourne, Victoria, Australia \\ ${ }^{7}$ Volunteer Services, Alfred Health, Melbourne, Victoria, Australia \\ ${ }^{8}$ Emergency Department, Cabrini Health, Malvern, Victoria, Australia \\ ${ }^{9}$ Volunteer Services, Cabrini Health, Malvern, Victoria, Australia \\ ${ }^{10}$ Nursing Services, Cabrini Institute, Cabrini Health, Malvern, Victoria, \\ Australia \\ ${ }^{11}$ Department of Mission and Strategy, Cabrini Health, Malvern, Victoria,
}

Contributors JAL conceived and developed the study protocol. JD and CB provided expertise with design of the intervention. All authors contributed to refinement of the study protocol. AL and JAL drafted the manuscript, and all authors approved the final manuscript.

Funding This work was supported by a grant from the Monash Partners Academic Health Science Centre.

Competing interests None declared.

Patient consent Obtained.

Ethics approval Alfred Health (432/15), Cabrini Health (12-09-11-15) and Monash University (CF15/4468-2015001934).

Provenance and peer review Not commissioned; externally peer reviewed.

Open Access This is an Open Access article distributed in accordance with the Creative Commons Attribution Non Commercial (CC BY-NC 4.0) license, which permits others to distribute, remix, adapt, build upon this work noncommercially, and license their derivative works on different terms, provided the original work is properly cited and the use is non-commercial. See: http:// creativecommons.org/licenses/by-nc/4.0/

\section{REFERENCES}

1. Lowthian JA, Curtis AJ, Jolley DJ, et al. Demand at the emergency department front door: 10-year trends in presentations. Med J Aust 2012;196:128-32

2. Lowthian JA, Jolley DJ, Curtis AJ, et al. The challenges of population ageing: accelerating demand for emergency ambulance services by older patients, 1995-2015. Med J Aust 2011;194:574-8.

3. Lowthian JA, Curtis AJ, Stoelwinder JU, et al. Emergency demand and repeat attendances by older patients. Intern Med $J$ 2013;43:554-60.

4. Lowthian JA, Stoelwinder JU, McNeil JJ, et al. Is the increase in emergency short-stay admissions sustainable? Trends across Melbourne, 2000 to 2009. Emerg Med Australas 2012;24:610-16.

5. Lowthian JA, Curtis AJ, Cameron PA, et al. Systematic review of trends in emergency department attendances: an Australian perspective. Emerg Med J 2011;28:373-7.

6. Aminzadeh F, Dalziel WB. Older adults in the emergency department: a systematic review of patterns of use, adverse outcomes, and effectiveness of interventions. Ann Emerg Med 2002;39:238-47.

7. Choi NG, Marti CN, Bruce ML, et al. Relationship between depressive symptom severity and emergency department use among low-income, depressed homebound older adults aged 50 years and older. BMC Psychiatry 2012;12:233.

8. Geller J, Janson P, McGovern E, et al. Loneliness as a predictor of hospital emergency department use. J Fam Pract 1999;48:801-4.

9. Shah MN, Jones CM, Richardson TM, et al. Prevalence of depression and cognitive impairment in older adult emergency medical services patients. Prehosp Emerg Care 2011;15:4-11.

10. Rottenberg Y, Jacobs JM, Stessman J. Depression and health service utilization from age 70 to 85 : the Jerusalem Longitudinal Study. J Am Med Dir Assoc 2013;14:711.e1-6.

11. Mistry R, Rosansky J, McGuire J, et al. Social isolation predicts re-hospitalization in a group of older American veterans enrolled in the UPBEAT Program. Unified Psychogeriatric Biopsychosocial Evaluation and Treatment. Int J Geriatr Psychiatry 2001;16:950-9.

12. McCusker J, Cardin S, Bellavance F, et al. Return to the emergency department among elders: patterns and predictors. Acad Emerg Med 2000;7:249-59. 
13. Sanders AB, Witzke D, Jones JS, et al. Principles of care and application of the geriatric emergency care model. In: Sanders AB, ed. Emergency care of the elder person. St Louis: Beverly Cracom Publications, 1996:59-93.

14. Hastings SN, Schmader KE, Sloane RJ, et al. Adverse health outcomes after discharge from the emergency departmentincidence and risk factors in a veteran population. $J$ Gen Intern Med 2007;22:1527-31.

15. McCusker J, Healey E, Bellavance F, et al. Predictors of repeat emergency department visits by elders. Acad Emerg Med 1997;4:581-8.

16. Victor $\mathrm{C}$, Scrambler S, Bond J, et al. Being alone in later life: loneliness, social isolation and living alone. Rev Clin Gerontol 2000;10:407-17.

17. Age UK. Later Life in the United Kingdom, January 2015 London. http://www.ageuk.org.uk/professional-resources-home/www.ageuk. org.uk/ (accessed June 2015).

18. Windle K, Francis J, Coomber C. Preventing loneliness and social isolation: interventions and outcomes. London: Social Care Institute for Excellence, 2011.

19. Office for National Statistics. Measuring national well-being-older people and loneliness, 2013. London: Office for National Statistics, 2013.

20. Franklin A, Tranter B. Loneliness in Australia. Housing and Community Research Unit-University of Tasmania, 2008.

21. National Ageing Research Institute. Beyondblue depression in older age: a scoping study. NARI, 2009.

22. Unutzer J, Katon W, Callahan CM, et al. Depression treatment in a sample of 1,801 depressed older adults in primary care. J Am Geriatr Soc 2003;51:505-14.

23. Hawton A, Green C, Dickens AP, et al. The impact of social isolation on the health status and health-related quality of life of older people. Qual Life Res 2011;20:57-67.

24. Holt-Lunstad J, Smith TB, Layton JB. Social relationships and mortality risk: a meta-analytic review. PLoS Med 2010;7:e1000316.

25. Nagayoshi M, Everson-Rose $\mathrm{SA}$, Iso $\mathrm{H}$, et al. Social network, social support, and risk of incident stroke: Atherosclerosis Risk in Communities study. Stroke 2014;45:2868-73.

26. Hawkley LC, Thisted RA, Masi CM, et al. Loneliness predicts increased blood pressure: 5-year cross-lagged analyses in middle-aged and older adults. Psychol Aging 2010;25:132-41.

27. James BD, Wilson RS, Barnes LL, et al. Late-life social activity and cognitive decline in old age. $J$ Int Neuropsychol Soc 2011;17:998-1005.

28. Shankar A, Hamer M, McMunn A, et al. Social isolation and Ioneliness: relationships with cognitive function during 4 years of follow-up in the English Longitudinal Study of Ageing. Psychosom Med 2013:75:161-70.

29. O'Luanaigh $\mathrm{C}, \mathrm{O}$ 'Connell $\mathrm{H}$, Chin $\mathrm{AV}$, et al. Loneliness and cognition in older people: the Dublin Healthy Ageing study. Aging Ment Health 2012;16:347-52.

30. Holwerda TJ, Deeg DJ, Beekman AT, et al. Feelings of loneliness, but not social isolation, predict dementia onset: results from the Amsterdam Study of the Elderly (AMSTEL). J Neurol Neurosurg Psychiatr 2014:85:135-42.

31. O'Connell $\mathrm{H}$, Chin AV, Cunningham $\mathrm{C}$, et al. Recent developments: suicide in older people. BMJ 2004;329:895-9.

32. Perissinotto CM, Stijacic Cenzer I, Covinsky KE. Loneliness in older persons: a predictor of functional decline and death. Arch Intern Med 2012;172:1078-83

33. Dinitz BS, Butters MA, Albert SM, et al. Late-life depression and risk of vascular dementia and Alzheimer's disease: systematic review and meta-analysis of community-based cohort studies. Br J Psychiatry 2013;202:329-35.

34. Ariyo AA, Haan M, Tangen CM, et al. Depressive symptoms and risks of coronary heart disease and mortality in elderly Americans. Cardiovascular Health Study Collaborative Research Group. Circulation 2000;102:1773-9.

35. Steptoe A, Shankar A, Demakakos P, et al. Social isolation, loneliness, and all-cause mortality in older men and women. Proc Natl Acad Sci USA 2013;110:5797-801.

36. Paul G, Smith SM, Whitford D, et al. Development of a complex intervention to test the effectiveness of peer support in type 2 diabetes. BMC Health Serv Res 2007;7:136.

37. Dale J, Caramlau IO, Lindenmeyer A, et al. Peer support telephone calls for improving health (Review). The Cochrane Collaboration, 2009.

38. Heisler M, Halasyamani L, Resnicow K, et al. 'I am not alone': the feasibility and acceptability of interactive voice response-facilitated telephone peer support among older adults with heart failure. Congest Heart Fail 2007;13:149-57.

39. Cattan M, Kime N, Bagnall AM. Low-level support for socially isolated older people - an evaluation of telephone befriending. In: Age UK, ed. Call in Time. London: Help the Aged, 2009:31-44.

40. Chapin RK, Sergeant JF, Landry S, et al. Reclaiming joy: pilot evaluation of a mental health peer support program for older adults who receive Medicaid. Gerontologist 2013;53:345-52.

41. Dale J, Caramlau I, Sturt J, et al. Telephone peer-delivered intervention for diabetes motivation and support: the telecare exploratory RCT. Patient Educ Couns 2009;75:91-8.

42. Rinaldi $\mathrm{P}$, Mecocci $\mathrm{P}$, Benedetti $\mathrm{C}$, et al. Validation of the five-item geriatric depression scale in elderly subjects in three different settings. J Am Geriatr Soc 2003;51:694-8.

43. Hughes ME, Waite LJ, Hawkley LC, et al. A short scale for measuring loneliness in large surveys: results from two population-based studies. Res Aging 2004;26:655-72.

44. Highet G, Crawford D, Murray SA, et al. Development and evaluation of the Supportive and Palliative Care Indicators Too (SPICT): a mixed-methods study. BMJ Support Palliat Care 2014:4:285-90.

45. Folstein MF, Folstein SE, McHugh PR. Mini-Mental State: a practical method for grading the cognitive state of patients for the clinician J Psychiatr Res 1975;12:189-98.

46. Leon AC, Davis LL, Kraemer HC. The role and interpretation of pilot studies in clinical research. J Psychiatr Res 2011;45:626-9.

47. EuroQol Group. EuroQol-a new facility for the measurement of health-related quality of life. Health Policy 1990;16:199-208.

48. Dennis CL. The process of developing and implementing a telephone-based peer support program for postpartum depression: evidence from two randomized controlled trials. Trials 2014:15:131.

49. Ritchie J, Spencer L. Qualitative data analysis for applied policy research. In: Bryman A, Burgess RG, eds. Analyzing qualitative data. London: Routledge, 1994:173-94.

50. Molloy GJ, McGee HM, O'Neill D, et al. Loneliness and emergency and planned hospitalizations in a community sample of older adults. J Am Geriatr Soc 2010;58:1538-41.

51. Coe RM, Wolinsky FD, Miller DK, et al. Elderly persons without family support networks and use of health services: a follow-up report on social network relationships. Res Aging 1985;7:617-22.

52. Dickens AP, Richards SH, Greaves CJ, et al. Interventions targeting social isolation in older people: a systematic review. BMC Public Health 2011;11:647.

53. Wheeler JA, KM G, Greenblatt B. The beneficial effects of volunteering for older volunteers and the people they serve: a meta-analysis. Int J Aging Hum Dev 1998;47:69-79. 